\title{
BUNCH LENGTH MEASUREMENTS AT THE TESLA TEST FACILITY USING A STREAK CAMERA
}

\author{
K. Honkavaara, Ph. Piot, S. Schreiber*, D. Sertore, DESY, 22603 Hamburg, Germany
}

\begin{abstract}
A streak camera provides a direct and convenient way to measure bunch lengths in the millimeter and submillimeter range. At the TESLA Test Facility (TTF) a streak camera with a subpicosecond resolution is in operation. A bunch compressor is used to produce bunch lengths down to $250 \mu \mathrm{m}$ for the operation of the TTF Free Electron Laser. Bunch length measurements are presented and compared with simulations.
\end{abstract}

\section{INTRODUCTION}

The TESLA Test Facility (TTF) has an injector based on a laser driven rf gun [1]. Its beam is used to test and to perform various experiments related to the TESLA linear collider project. Among this, it serves as an injector to drive the TTF-FEL free electron laser [2]. The injector is followed by accelerating module 1 , a magnetic chicane bunch compressor (BC2) [3], and a second accelerating module 2 . A spectrometer dipole magnet situated in the experimental area at the end of the linac is used for energy measurements. A sketch of the linac is shown in Fig. 1.

To drive the FEL, high peak current and short bunch lengths are important parameters. This report presents bunch length measurements using a streak camera with a resolution below $1 \mathrm{ps}$ (sigma). Since this streak camera cannot resolve the compressed bunch of $250 \mu \mathrm{m}$ the primary goal of the measurement was to measure and understand the longitudinal bunch form produced by the injector and to have an upper limit on the compressed bunch length. Effects of the rf gun phase and the phase of the first acceleration module on the bunch length have been studied.

\section{EXPERIMENTAL SET-UP}

We use synchrotron radiation (SR) produced by the horizontally deflecting spectrometer dipole after acceleration (see Fig. 1) The light is guided by a set of $\mathrm{Al}$ mirrors through a $11 \mathrm{~m}$ long transport line to the streak camera [4]. The entrance slit of the camera is oriented horizontally, i.e. in the same plane as the dipole. In our case, the effect of the horizontal spread of the SR fan on the time resolution is negligible. The slit is imaged onto the photocathode; its function is to reduce the transverse dimension of the image. The photocathode used (S-20) is sensitive for wavelengths between 200 and $800 \mathrm{~nm}$. The input optics is adapted to this wavelength range. This allows measurements of the

\footnotetext{
*Email: siegfried.schreiber@desy.de
}

UV pulses of the photocathode drive laser ( $262 \mathrm{~nm}$ wavelength) as well. For the SR experiments, an additional focusing achromat lens is used, which has a cut off at $350 \mathrm{~nm}$. A CCD camera used to record the streak images.

The maximum streak speed is $10 \mathrm{ps} / \mathrm{mm}$ providing a resolution of $0.25 \mathrm{ps}$ per CCD pixel. The intrinsic time resolution of the streak camera tube depends on the wavelength: the measured values (sigma) for the maximum streak speed are $0.55 \pm 0.09 \mathrm{ps}$ for $\lambda=760 \mathrm{~nm}$ and $0.9 \pm 0.13 \mathrm{ps}$ for $\lambda=$ $380 \mathrm{~nm}$.[5] Due to the low intensity of the light signal, the whole wavelength range from 350 to $800 \mathrm{~nm}$ had to be used in this experiment. Since the spectral response is roughly symmetric in this wavelength range, we use for the maximum streak speed an average resolution of $\sigma_{\text {res }}=0.7 \mathrm{ps}$.

\section{MEASUREMENTS}

The electron bunch length has been measured in two experiments for various phase settings of the rf gun and the accelerating modules. In addition, the pulse length of the photocathode laser has been measured as well. In the first experiment, the electron bunch length of the injector versus the relative phase between the rf gun and the laser (gun phase) has been measured. To do this, the beam has been transferred straight through the linac without using the bunch compressor $\mathrm{BC} 2$. We assume, that in this case the bunch length does not change during transport and acceleration on crest of both modules. In the second experiment, the compression of the electron bunch with $\mathrm{BC} 2$ has been measured as a function of the phase of the first accelerating module. The bunch compressor $\mathrm{BC} 1$ has not been used in these experiments.

\section{DATA ANALYSIS}

In order to fully exploit the resolution of the camera, the entrance slit of the camera has to be small. For the fastest streak speed, a slit width of $100 \mu \mathrm{m}$ corresponds to a resolution of 1 ps. An image of the slit has been taken during every experiment. In the first experiment the slit size was $\sigma_{\text {slit }}=110 \mu \mathrm{m}$. In the second experiment, the slit was larger to allow more light intensity on the camera: $\sigma_{\text {slit }}=$ $190 \mu \mathrm{m}$.

In this analysis, we assume that the resolution is dominated by the streak tube resolution and the slit size. Contributions from other sources like the CCD camera pixel size and diffraction effects are small compared to that. The intrinsic tube resolution and the slit width are quadratically subtracted from the measured value.

For every machine setting, several images are taken. The 


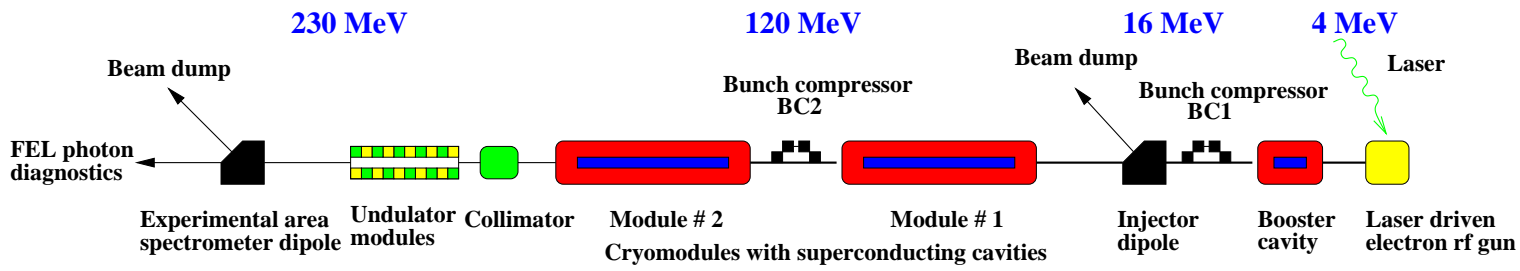

Figure 1: Schematic layout of the TTF linac (not to scale, total length about $100 \mathrm{~m}$ ). Approximate beam energies are indicated.

projection of the image onto the time axis is fitted by a gaussian function with a weighting of 1 per pixel, including a constant background. The bunch length is then considered as the mean value of the widths (sigma) of all profiles. The error is estimated as the rms variance.

In order to calibrate the gun phase, the transmitted charge through the gun is measured as a function of the relative phase between gun and laser (phase scan). Phase zero is the phase, where half of the charge is transmitted. In addition, the laser pulse length can be estimated from the rising edge of the scan.[6] The phase of the gun and of the accelerating modules are stabilized by a feedback system. A phase value can be set with a relative accuracy of better than $1^{\circ}$ of $1.3 \mathrm{GHz}$.

\section{RESULTS}

Examples of the laser pulse profile and longitudinal bunch profiles are shown in Fig. 2. Upper left profile is an example of laser pulse ( $\left.\sigma_{\mathrm{t}}=8.5 \mathrm{ps}\right)$, and the next two profiles (upper right, lower left) represent an uncompressed bunch at the nominal gun phase of $40^{\circ}\left(\sigma_{\mathrm{z}}=3.4 \mathrm{~mm}\right.$ and $2.8 \mathrm{~mm}$, resp.). The lower right profile is for maximum compression and has a length of $1.1 \mathrm{~mm}$.

Figure 3 shows the injector bunch length as a function of the gun phase.

As expected, the bunch length decreases with decreasing gun phase. This has also been observed in previous streak camera measurements at TTF [7] as well as at the A0 test stand at Fermilab [8]. The data are compared to simulations with the codes ASTRA [9] and PARMELA [10] for different laser pulse lengths. ASTRA favors a laser pulse length of $12 \mathrm{ps}$, while for PARMELA 14 ps fits best to the data.

The prediction by the codes for phases above $50^{\circ}$ differs. This difference may be explained by the fact that ASTRA simulations have been made up to the entrance of the booster cavity, therefore accounting some bunch lengthening due to space charge forces in the drift space, while PARMELA simulations end at the gun exit. Despite this, the agreement between measurements and simulations is good for phases between 0 and $50^{\circ}$. Unfortunately, measurements for phases above $60^{\circ}$, where the codes differ most, have not been possible.

The laser pulse length has been measured with the same streak camera. A gaussian fit on the measured profiles
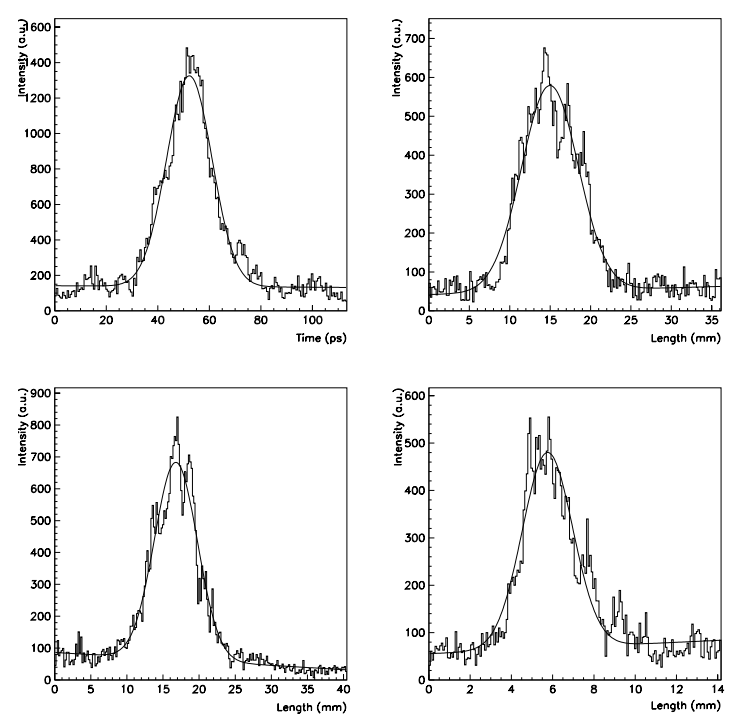

Figure 2: Examples of a laser pulse profile (upper left) and longitudinal bunch profiles without (upper right, lower left), and with bunch compression (lower right). A gaussian fit on profiles is shown.

yields a laser pulse length of $\sigma_{\mathrm{t}}=8.8 \pm 0.6 \mathrm{ps}$. This is in fair agreement with the simulations.

Figure 4 shows the effect of bunch compression using $\mathrm{BC}$ 2: the bunch length is plotted for different phases of the accelerating module 1 . In these measurements the gun is operated close to the nominal phase, and module 2 is on crest. Maximum compression of the bunch to $\sigma_{\mathrm{z}}=$ $1.0 \pm 0.1 \mathrm{~mm}$ has been measured for a module 1 phase of $-10^{\circ}$ corresponding $-16^{\circ}$ off crest acceleration. Simulations performed with ASTRA and an analytical model of the compressor are in agreement with the data (see Fig. 4).

Although the rms bunch length predicted by simulations is in the mm-range, the simulations also predict that the bunch charge density consists of a spike containing half of the charge, and a long tail. This results from non-linear effects in the bunch compressor. This peak has been observed with other methods (see $[11,12]$ ). We hope to be able to resolve this predicted peak in the near future with a faster streak camera purchased recently.

We also tried to find substructures in the electron bunch or in the laser pulse itself. This has been motivated to explain the beam break-up phenomena observed in the energy 


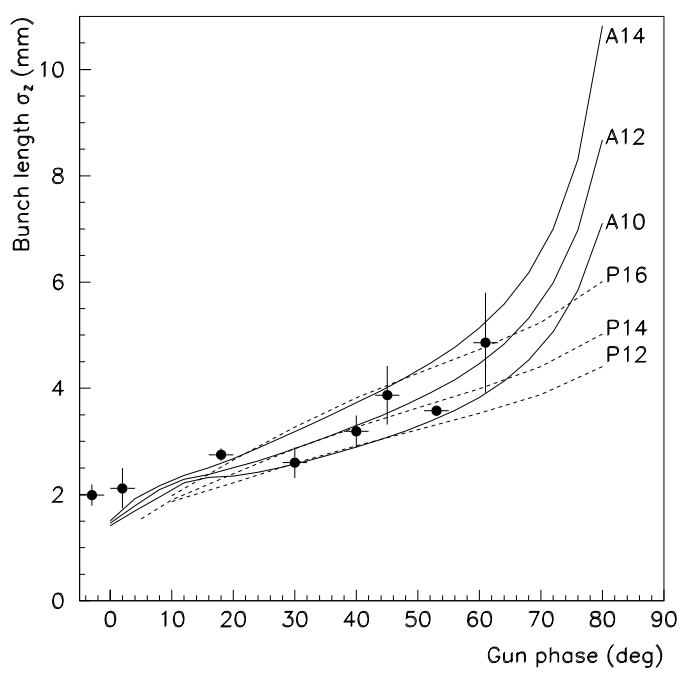

Figure 3: Bunch length without bunch compression vs. gun phase. Results of simulation by ASTRA (A) and PARMELA $(\mathrm{P})$ are shown for different laser pulse lengths. The number indicates the laser pulse length in ps (sigma).

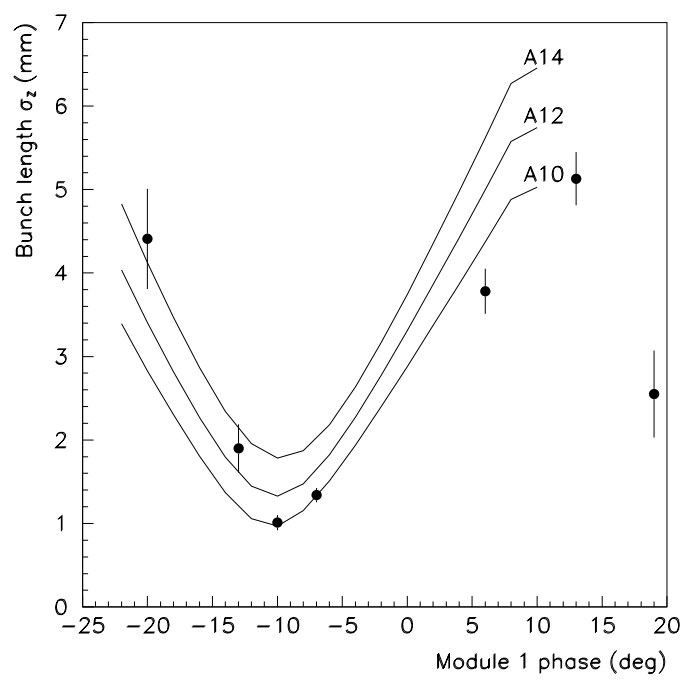

Figure 4: Bunch length vs. phase in accelerating module 1 using the bunch compressor $\mathrm{BC} 2$ compared to simulations with ASTRA. The number indicates the laser pulse length in ps (sigma).

space at full compression.[11, 12] From our measurements, it was not possible to identify longitudinal substructures, neither in the electron bunch, nor in the laser pulse. In some profiles, spikes are visible. However, these spikes are most likely due to statistical fluctuations of the photon signal. The total number of photons arriving at the camera is only in the order of $10^{4}$, so that the number of photons per pixel on the CCD camera is low and subject to strong statistical fluctuations.

\section{CONCLUSION}

The bunch length decreases as expected with decreasing gun phases. The smallest bunch length measured for maximum compression is $\sigma_{\mathrm{z}}=1.0 \pm 0.1 \mathrm{~mm}$. The measurements were not limited by the resolution of the camera. The data are also in agreement with simulations.

\section{ACKNOWLEDGEMENT}

We like to thank T. Garvey, B. Leblond, and M. Bernard from Laboratoire de l'Accélérateur Linéaire (LAL), Orsay, France for making the streak camera available for these measurements.

\section{REFERENCES}

[1] S. Schreiber, "Performance Status of the RF-gun based injector of the TESLA Test Facility Linac", Proc. of EPAC2000, Vienna, Austria, p. 309.

[2] J. Andruszkow et al., "First Observation of Self-Amplified Spontaneous Emission in Free-Electron Laser at $109 \mathrm{~nm}$ Wavelength”, Phys. Rev. Lett. 85 (2000) 3825-3829.

[3] M. Geitz et al, "Bunch Compressor II at the TESLA Test Facility”, Proc. of PAC'99, New York, p. 2507.

[4] On loan from LAL, Orsay, France; manufactured by ARP, now Photonetics, D-77694 Kehl, Germany.

[5] "Résultats de Test Caméra à Balayage de Fente aprés l'installation du tube type FSO", ARP/PSU 4/7/95.

[6] S. Schreiber et al, "Measurement of Space Charge Effects and Laser Pulse Length in the TTF RF Gun using the Phase Scan Technique", Proc. 21st Int. Free Electron Laser Conf., August 1999, Hamburg, Germany, p. II-69.

[7] M. Geitz et al., "Determination of the Longitudinal Phase Space Distribution produced with TTF Photo Injector", Proc. of the 21st Int. Free Electron Laser Conf., August 1999, Hamburg, Germany, p. II-83.

[8] J.-P. Carneiro, "Etude expérimentale du photo-injecteur de Fermilab", PhD-Thesis, Univ. de Paris-Sud, Orsay, France.

[9] K. Flöttmann, ASTRA user manual, www.desy.de/ ${ }^{\sim}$ mpyflo/ASTRA_dokumentation.

[10] PARMELA, Version 5.0, B. Mouton, LAL, F-91405 Orsay.

[11] M. Hüning, "Recent Results from the Tesla Test Facility (TTF)", WOPA012 this conference.

[12] M. Hüning, "Investigations of Longitudinal Charge Distribution in very short Electron-bunches", Proc. 5th European Workshop on Diagnostics and Beam Instrumentation May 2001, Grenoble, France. 